\title{
NEW DIVISIBILITY TESTS
}

\section{Pagdame Tiebekabe and Ismaïla Diouf}

Université Cheikh Anta Diop (UCAD), Laboratoire

d'Algèbre, de Cryptologie, de Géométrie Algébrique et

Applications (LACGAA)

Dakar, Sénégal

\begin{abstract}
In this article, we first made the inventory of some various divisibility tests of 7 known, studied the effectiveness of these tests, demonstrate the new divisibility test of 7 proposed by the young Nigerian Chika Ofili and an algorithm in \#Python. Secondary, we proposed and demonstrated a new test of divisibility by 3 which we called in this paper Diouf-Pagdame Test based mainly on the calculation of $d-2 e$ where $d$ and $e$ represents respectively digits of tens and units of any given number. We have also emphasized the educational aspect of these methods.
\end{abstract}

\section{Introduction}

The divisibility of a number by 2 or 5 is very simple. It is known that a number $N$ is divisible by 2 if its last digit is an even integer and is divisible by 5 if its last digit is 0 or 5 . A number $N$ is divisible by 3 if the sum of its digits is divisible by 3 . However, these three tests can be easily explained using modulo arithmetic.

In this paper, we only deal with the case of the divisibility by 7 test and

Received: January 8, 2021; Accepted: January 25, 2021

Keywords and phrases: Divisibility test, Congruence, Numerations, Algorithms. 
briefly discuss the 3-divisibility test. The 7-divisibility test has drawn the interest of some researchers. This gave birth to several techniques of divisibility. However, some tests have been proven more effective than others have, especially when it comes to verify the divisibility by 7 of a large number. In fact, if the divisibility of some numbers seems easy, it is however not the case of the test divisibility by 7 . Some tests allow it to be done easily with few lines whereas for others, we need a lot of pages. It is in this thought that the recent discovery of a Nigerian youth Chika Ofili has caused a lot of ink in the newspapers, which had allowed him to win a British prize for the discovery of a new mathematical formula. Before, its discovery there were many existence tests. Among those tests, we have the Toja's test, the property of multiples of 7 , the criterion for a large number, the method of utilization of a diagram, the criterion Pascal divisibility, and so on. These new tests of divisibility can be used for educational purpose due to their simplicity and efficiency. Therefore, we think we can start using them earlier in school environment.

In this article, we will list some known tests, demonstrate the divisibility test of 7 proposed by Chika, state and demonstrate a new divisibility test of 3 that we have discovered, compare these tests and determine the most effective of these tests by comparing the execution times of different algorithms in Python of each methods.

\section{Historical Background}

We can define a divisibility test as an algorithm, which uses the digits of an integer $N$ to determine whether $N$ is divisible by a divisor $d$. The history of divisibility tests started back from at least 500 C.E. when a divisibility test for 7 was included in the Babylonian Talmud. It was from then that methods which provide divisibility tests for all positive integers have been discovered and rediscovered by different mathematicians such as Joseph-Louis Lagrange [10], and Charles Dodgson (a.k.a. Lewis Carroll, author of Alice In Wonderland) [8]. A remarkable summary of the literature in relation to divisibility tests published prior to 1915 is provided in Leonard Dickson's History of the 
Theory of Numbers [3]. Edward Brooks dedicated two chapters to the study of divisibility tests in his 1880 book titled The Philosophy of Arithmetic [2]. Recently in (2006), Marc Renault published a wonderful article [11] that provides divisibility tests for all integers between 2 and 102, which includes brief explanations for why the tests work. And lastly, Eric L. McDowell (Berry College) in Divisibility Tests: A History and User's Guide presents a modest survey of some of the more recent literature regarding divisibility tests.

\section{Some Existing Divisibility Tests of 7}

\subsection{Property of Multiples of 7}

There are already some effective methods that will allow to verify the divisibility of a given number. Among those tests, we can cite property of multiples of 7. The principle is quite simple. This test actually consists of verifying if the difference of the tens digit by the double of the units is a multiple of 7 . If that difference is a multiple of 7 , then the number to be tested is also a multiple of 7.

\subsection{Criterion for a large Number}

A second method based only on the fact that 103 is congruent to -1 modulo 7, is to separate this number in 3-digit slices starting from the units and insert alternately - and + between the slices. We carry out the operation thus written and this result is divisible by 7 if and only if the starting number was.

Example Let us take the number 5527579818992. We will separate it into three-digit sections starting from the units:

$$
5 \text { | } 527 \text { | } 579 \text { | } 818 \text { | } 992 .
$$

We interpose alternately - and +:

$$
5-527+579-818+992 .
$$

We carry out the operation thus written: 


$$
5-527+579-818+992=231 .
$$

We see if 231 is divisible using the divisibility lemma by $7: 23+5 * 1=28$ is divisible by 7 so 5527579818992 is.

Proof. Since $1000 \equiv-1(\bmod 7)$ and $1000000 \equiv 1(\bmod 7)$, then for all integer $n>0$, we have: $\left(10^{3}\right)^{2 n} \equiv 1(\bmod 7)$ and $\left(10^{3}\right)^{2 n-1} \equiv-1(\bmod 7)$. Any integer $N$ can be written in the form:

$a \times\left(10^{3}\right)^{2 n}+b \times\left(10^{3}\right)^{2 n-1}+\ldots+\times\left(10^{3}\right)+y \times 10^{3}+z$. Hence we can directly conclude that $N \equiv 0(\bmod 7)$ if $z-y+x-\ldots-b+a \equiv 0(\bmod 7)$.

\subsection{The Toja's Method}

This third method is similar to the second one based only on the fact that $10^{3}$ is congruent to -1 modulo 7 , from which we deduct that if si

$$
x=100^{m} b_{m}+\ldots+100^{2} b_{2}+100^{1} b_{1}+b_{0}
$$

and

$$
y=10^{m} b_{0}-10^{m-1} b_{1}+10^{m-2} b_{2} \ldots(-1)^{m} b_{m}
$$

then

$$
10^{m} x \equiv y(\bmod 7)
$$

Therefore, $x$ is divisible by 7 if and only if $y$ is. We can definitely replace by the way every $b_{i}$ by any integer, which is congruent to modulo 7 . The principle is therefore to cut the number $x$ into slices of 2 digits and find the distance between each number of 2 digits and the nearest multiple of 7 (alternately by excess and by default).

Example 2.3. Let us take the number 5527579818992. We will separate it into two-digit sections starting from the units 5 | 52 | 75 | 79 | 81 | 89 | 92. 
- From the right, the nearest multiple of 7 by default is 91 : the distance $92-91=1$;

- For the second pair, the nearest multiple of 7 by excess is 91: the distance $91-89=2$;

- For the third pair, the nearest multiple of 7 by default is 77 : the distance $81-77=4$;

- For the fourth pair, the distance: $84-79=5$, and so on.

The starting number is a multiple of 7 if and only if $1|2| 4|5| 5|4| 5$ is a multiple of 7 (The different "remainders" are writtens in reverse order). We also notice that the divisibility by 7 of 1245545 is equal to that of 3136, and of 14 , therefore 5527579818992 is divisible by 7 .

\subsection{Method of utilization of a diagram}

This technique is based [2] on the writing of a number in base 10 and on the modulo 7 congruence. The use of a diagram was proposed in 2009 by David Wilson [3, 4]. On a circle, we have all the numbers from 0 to 6, i.e. all possible modulo 7 remainders. We then match with an arrow each remainder $r$ with the rest modulo 7 of $r * 10$.

The diagram is then used as follows: for the integer: $\overline{a_{n} a_{n-1} \ldots a_{1} a_{0}}$ equal to

$$
\left(\ldots\left(\left(a_{n} * 10+a_{n-1}\right) * 10+a_{n-2}\right) * 10+\ldots\right) * 10+a_{0} .
$$

- We stand on the case 0 and we are moving on the circle of $a_{n}$ cases. We then obtain the remainder of an modulo 7;

- We then borrow the arrow, which starts from the square where we are and, from the point of arrival of the arrow, we move on the circle of $a_{n-1}$ boxes. We then obtain the remainder of $a_{n} * 10+a_{n-1}$ modulo 7;

- We then repeat the process (borrowing an arrow, and then moving on the circle) until $a_{0}$. 
The number is divisible by 7 if and only if the arrival box is box 0 .

This figure is:

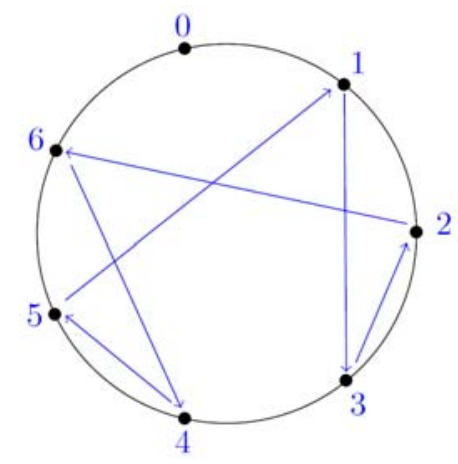

Standard and we move on the boxes according to the procedure stated previously.

Example 2.4. Suppose we want to verify the divisibility by 7 of 4564 . Here $a_{0}=4, a_{1}=6, a_{2}=5$ et $a_{3}=4$.

- We move from 0 to $a_{3}=4$ boxes and we arrive at 4 ;

- We take the arrow which leads from 4 to 5 . We move from $a_{2}=5$ boxes and we arrive at 3 ;

- We take the arrow which leads from 3 to 2 . We move from $a_{1}=6$ boxes and we arrive at 1 ;

- We take the arrow which leads from 1 to 3 . We move from $a_{0}=4$ boxes and we reache 0 .

The arrival box being 0 , then the number 4564 is a multiple of 7 .

\subsection{Pascal's Criterion of divisibility}

We divide 1 by 7 , and we write at each stage of the calculation the successive remains. We obtain: $\ldots, 5,4,6,2,3$ and 1 , if we reverse the order.

This number is called Pascal's key. 
With the number to be tested, we multiply the number of units by 1 , the tens digit by 3 , the hundreds by 2 , and so on. Finally, we add the results obtained. If the number obtained is a multiple of 7 , then the initial number to be tested is a multiple of 7 .

Example 2.5. 644 is a multiple of 7, because $4 \times 1+4 \times 3+6 \times 2=28$ which is a multiple of 7 . So therefore, 644 is also a multiple of 7 .

\subsubsection{Proof of Pascal's criterion}

If we perform the division of 1 by 7 , we obtain $0 ; 142857$ and a sequence of remains in the division: $1,3,2,6,4,5, \ldots$

For example, for the first decimal places of $0 ; 142857$, we have

$$
\begin{aligned}
& 7 \times 0+1=10^{0} \\
& 7 \times 1+3=10^{1} \\
& 7 \times 14+2=10^{2} \\
& 7 \times 142+6=10^{3} \text { etc... }
\end{aligned}
$$

this table is presented as a bridge which links base 10 and base 7.

Let $r_{n}$ the rest of the division for the $n^{\text {th }}$ power of 10 and $d_{n}$, the number $1 / 7$ estimated at $n$ decimal places multiplied by $10^{n}$.

We have then $7 \times d_{n}+r_{n}=10^{n}$ which is a generalisation of the precedent table.

Let $N$ a number of $n$ digits such that $N=N_{n} \times 10^{n}+N_{n-1} \times 10^{n-1}$ $+\ldots+N_{1} \times 10^{1}+N_{0}$ with $N_{i} \in[0,9]$ and $N_{i} \in \mathbb{N}$.

By applying the calculation suggested by the criterion, we obtain $N_{0}(7 \times 0+1)+N_{1}(7 \times+3)+\ldots+N_{n}\left(7 \times d_{n}+r_{n}\right)=N_{0}+N_{1}(7 \times 1)+N_{1} \times 3$ $+\ldots+7 N_{n} d_{n}+N_{n} r_{n}$. The first terms of each parenthesis being multiples of 
7, we can ignore them. If $N=N_{n} \times 10^{n}+N_{n-1} \times 10^{n-1}+\ldots+N_{1} \times 10^{1}+N_{0}$ is a multiple of 7 , then $\sum N_{i}\left(7 d_{i}+r_{i}\right)$ (which by hypothesis is equal to the previous expression must be divisible by 7 for little $\sum m_{i} r_{i}$ so are.

\section{New Divisibility Tests}

Statement of Chika's divisibility test

Let $d, e$ be non-negative integers, $e \leq 9$ and $m=10 d+e$. Thus $7|m \Leftrightarrow 7| d+5 e$.

Here we give a proposal of a new theorem.

Theorem (Diouf-Pagdame). Let $d$, e be non-negative integers, $e \leq 9$ and $m=10 d+e$. Then $3|m \Leftrightarrow 3| d-2 e$.

\section{Proof of Theorems}

We will prove the two theorems together. Let $m$ be the number to be tested. $m$ is written in the form of $m=10 d+e$ where $e$ is the last digit and $d$ is the remainder of the number. $m$ can also be written in the form $m=10 d+$ $e-3(d+5 e)+7(d-2 e)$. With $m$ in this form, we can remark that, $m=3(d+5 e)+7(d-2 e)$ is divisible by 3 , since $(3,7)=1$, if and only if $3 \mid(d-2 e)$. Similarly $m=3(d+5 e)+7(d-2 e)$ is divisible by 7 if and only if $7 \mid(d+5 e)=c \cdot c(d+5 e)$ is known as "Chika's number".

This ends the proof.

\section{Main Results and Observations}

\subsection{Main results}

In this section, we present the execution time of the different programs on ASUS CORE i5 8th Gen. For a sufficiently given large number, we evaluate the Python code of the various tests listed in this article, and we note the execution time in a summary tables. We have chosen two numbers, one 
of which is divisible by 7 and the other is not. This in order to verify the effectiveness of the various tests. These numbers are

$N_{1}=92345677890981132537784986527836547577827648576535125361$ 23456789098760774 and

$N_{2}=34526780209364787384690124356272134567890272134563767471$ 23456789723427237.

Let's called

Method 1: Property of multiple of 7;

Method 2: Criterion for a large number;

Method 3: Toja's method;

Method 4: Method of utilization of diagram and

Method 5: Pascal's method.

Below are the tables of the execution times of the different methods.

Table 1. For $N_{1}=923456778909811325377849865278365475778276485$ 7653512536123456789098760774

\begin{tabular}{|c|c|c|c|}
\hline Methods & Method 1 & Method 2 & Method 3 \\
\hline Time of executions & 0:024769759000264457 & 0:024846616999866455 & 0:024533451000024797 \\
\hline
\end{tabular}

\begin{tabular}{|c|c|}
\hline Method 4 & Method 5 \\
\hline 0:022657221000145 & $0: 020596608999994714$ \\
\hline
\end{tabular}

Table 2. For $N_{2}=34526780209364787384690124356272134567890272$ 134563767471234567897234272345

\begin{tabular}{|c|c|c|c|}
\hline Methods & Method 1 & Method 2 & Method 3 \\
\hline Time of executions & $0: 022463982999852306$ & $0: 023879767999915202$ & $0: 02267362900010994$ \\
\hline \\
\cline { 2 - 4 } \\
\cline { 2 - 4 } & Method 4 & Method 5 \\
\cline { 2 - 4 }
\end{tabular}

\subsection{Remarks}

By the help of Python programs on the different tests, we can compare 
them by using the execution time as a parameter of comparison. Indeed, we randomly chose a number large enough (of the order of 60 digits) to test the programs. As the tables above indicate, the differences appear to be negligible. however small it is, makes all the difference between the different methods. We have kept several digits after the comma to be as precise as possible. The difference in execution time between Method 5 (the most efficient) with the lowest execution time and Method 2 (the least efficient) with the longest time is 0:004250008 and 0:006796797 from Table 1 and Table 2 respectively. In view of these results, taking the execution time as a comparison parameter, method 5 seems to be the most efficient and method 2 is the least efficient among those methods which have been studied.

\section{Conclusion}

Finally, we will conclude that these methods are all very efficient. However, some are more efficience than others in their simplicity. Simple in their understanding and execution. According to the execution times, Pascal's criterion is the most efficient among the tests of divisibility by 7. Pascal's criterion is most efficient in view of the results obtained in the section 6 but is little too heavy. It is based on interesting property which emerges in the passage from base 7 to decimal base. It uses a sequential digit key. But its inconvenience side is usefulness. It is advantageous when we deal with several numbers.

Hence, we should highlight many similarities between the tests. Between the method of property of multiples of 7 and Diouf-Pagdame test, we can notice the use of the number $d-2 u$ in the two cases. The methods "criterion of large number" and the "Toja's method" have in common the separation by slices from the units. The first by the slice of 3 and the second by the slice of 2.

\section{References}

[1] The Moscow Puzzles: 359 Mathematical Recreations, Dover Publications, p. 140, 2014 (1re éd. 1971). 
[2] Edward Brooks, The Philosophy of Arithmetic. Normal Publishing Company, 1880.

[3] Leonard Eugene Dickson, History of the Theory of Numbers, Chelsea Publishing Company, (Originally published in 1919 by the Carnegie Institution, Washington, D.C., all three volumes of this text are now available in paperback from Dover Publications, Mineola, NY, 2005.), 1952.

[4] Babylonian Talmud, Abod. Zar. 9b.

[5] Y. Cherniavsky and A. Mouftakhov, Zbikowskis Divisibility Criterion, The College Mathematics Journal 45(1) (2014), 17-21.

[6] L. E. Dickson, History of the theory of numbers, Vol. I: Divisibility and primality, Chelsea Publishing Co., New York 1966.

[7] J. L. Lagrange, Leçonns élém. sur les math. données à l'école normale en 1795, Jour. de l'école polytechnique 7, 8, 194-9; Oeuvres, 7, pp. 203-8, 1812.

[8] Charles L. Dodgson, Brief method of dividing a given number by 9 or 11, Nature, 56 (1459), 565-566, 1897.

[9] A. Zbikowski, Note sur la divisibilité des nombres, Bull. Acad. Imp. Sci. SaintPétersbourg 3 (1861), 151-153.

[10] Joseph-Louis Lagrange, Leçons élémentaires sur les math. données á l'école normale en 1795, Journalde l'école polytechnique 7,8:194-199, 1812.

[11] Marc Renault, Stupid divisibility tricks: 101 ways to stupefy your friends, Math Horizons 14(2) (2006), 18-21, 42. 
Paper No. PPH-2101019-ME

Kindly return the proof after correction to:

The Publication Manager

Pushpa Publishing House

Vijaya Niwas

198, Mumfordganj

Prayagraj-211002 (India)

along with the print charges*.

*Invoice attached
Proof read by:

Copyright transferred to the Pushpa Publishing House

Signature:

Date:

Tel:

e-mail:

Number of additional reprints required

Cost of a set of 25 copies of additional reprints @ U.S. Dollars 15.00 per page.

(25 copies of reprints are provided to the corresponding author ex-gratis) 